\title{
Effect of Integrated Nutrient Management on Plant Growth and Yield of Rabi Maize under Irrigated Conditions of Ajmer
}

\author{
Rubina Gill ${ }^{1}$, Prasoon Singh $^{2}$, Rajesh Kumar ${ }^{3}$ and Bijendra Kumar ${ }^{4 *}$ \\ ${ }^{1}$ Innocent Hearts Group of Institutions, Punjab Technical University, \\ Jalandhar (Punjab) 144026, India \\ ${ }^{2}$ Sriram Fertilizer and Chemical Ltd. Ludhiana, India \\ ${ }^{3}$ DAV College Ajmer Rajasthan, Swami Keshwanand Rajasthan Agricultural University \\ 334006, India \\ ${ }^{4}$ Department of Genetics and Plant Breeding, School of Agriculture, Lovely Professional \\ University, Phagwara (Punjab) 144401, India \\ *Corresponding author
}

\section{A B S T R A C T}

Keywords

Biofertilizer,

FYM, INM,

Vermicompost,

VAM

Article Info

Accepted:

16 February 2018

Available Online:

10 March 2018
Afield experiment was conducted during the Rabi (winter) season of 2012 and 2013, at Dayanand College Ajmer, to study the response of maize (Zea mays L.) variety 'Ganga safed -2 ' to integrated use of organic, inorganic and biofertilisers. The experiment was laid out in randomized block design with sixteen treatments and three replications. Different levels of inorganic $(60 \mathrm{~kg} / \mathrm{ha}$ and $120 \mathrm{~kg} / \mathrm{ha} \mathrm{N})$ and organic (poultry manure, vermi compost and FYM) were applied with or without VAM, Azotobactor and Azospirillium inoculation. The results revealed that the combination of Azotobactor + vermicompost $+60 \mathrm{~kg} / \mathrm{ha} \mathrm{N}$ (urea) gave the best results and showed significant improvement in growth, yield attributing characters and seed yield (53.56) q/ha than other treatment combinations.

\section{Introduction}

Maize is the third most important cereal crop with a wide adaptability and grown throughout the world for grain and fodder which is referred to as the "Queen of cereals". Cultivation during winter is becoming a common practice in Penninsular India (Andhra Pradesh, Karnataka and Tamil Nadu) as well as in North- Eastern plains. It has the potential to yield as $10-12$ tonnes / hectare and possibilities of further increase in productivity substantially up to 18 tonnes with better management . The demand of maize is expected to double worldwide by 2050. Maize is grown on 11.5 lakh hectare area in Rajasthan state and majority of production is taken from south Rajasthan. Yield components including number and weight of grains/cob, harvest index(HI), grain 
filling period (GEP), grain yield and physiological parameters including LAI,LAB,TDM and plant nitrogen content in winter maize than in monsoon crop (Kumar and Singh, 1999).

Among the several causes, improper nutrient management is the one for low productivity. Hence it has become the need of hour to enhance the productivity of winter maize by adapting feasible scientific and sustainable nutrient management practices. A single nutrient source cannot alone meet the complete plant nutritional demand. The continuous use of high levels of chemical fertilizers is adversely affecting the sustainability of agricultural production and causing environmental pollution (Virmani, 1994). Since organic manures cannot meet the total nutrient needs of modern agriculture, integrated use of nutrients from the fertilizers and organic sources seems to be need of the time. Maize being a heavy feeder of nutrients requires a good quality of nutrients. Integrated nutrient management has a great potential to off-set the growing heavy nutrient demands, to achieve maximum yields and to sustain the crop productivity on long term basis. More technically integrated nutrient management system refers to an approach in which the nutrient needs of a crop are met from the approximately combined use of fertilizers, crop residues, recyclable wastes, organic manures and bio-fertilizers. This experiment was conducted to work out the suitable nutrient management system to enhance the yield and productivity of rabi maize in Ajmer region of Rajasthan.

\section{Materials and Methods}

The experiment was conducted during two consecutive years 2012 and 2013 of rabi season at crop research farm, Dayanand college Ajmer, Rajasthan. The soil of the experimental field was sandy loam with available nitrogen $118 \mathrm{~kg} / \mathrm{ha}$, phosphorus 50 $\mathrm{kg} / \mathrm{ha}$ and potash $233 \mathrm{~kg} / \mathrm{ha}$. The $\mathrm{pH}$ of soil was 7.4 and the organic carbon was $0.27 \%$. The experiment was laid out in randomized block design with sixteen treatments and three replications. The variety 'Ganga safed- 2' was sown during 2012 and 2013 in month of October with a spacing of $60 \mathrm{~cm} \times 25 \mathrm{~cm}$ and plot size $3 \mathrm{~m} \times 2 \mathrm{~m}$. The recommended dose of fertilizers for the crop was $120-80-60 \mathrm{~kg} / \mathrm{ha}$ $\mathrm{N}, \mathrm{P}_{2} \mathrm{O}_{5}$ and $\mathrm{K}_{2} \mathrm{O}$. Two levels of inorganic 50 $\%$ and $100 \%$ NPK and three organic (vermicompost, farmyard and poultry manure) treatments with or without Azotobacter, VAM and Azospirillium inoculation as per their set levels in the treatment combination were applied and mixed thoroughly with the soil. Half of nitrogen and entire dose of phosphorus and potash was applied as basal dressing to the plots before sowing. The remaining nitrogen was applied in two equal splits, one half at knee height stage of the plant and the remaining at tassel initiation stage as topdressing, poultry manure, FYM and vermicompost as per their set levels in the treatment combination were applied and mixed thoroughly with the soil before one week. Organic manures are useful to enhance the physical as well as chemical properties of soil. Organic matter acts directly as a source of plant nutrients and indirectly influence the physical and chemical properties Kumar and Singh (1999). Organic manures benefits by giving positive results such as, increased plant vigor, less nutrient requirement through chemical fertilizer, increased water holding capacity of soil, increased crop production.

Biofertilisers are being essential component of organic farming. Azospirillium is an associative symbiotic nitrogen fixing bacteria and seed treatment has been given @ $250 \mathrm{~g} /$ $10 \mathrm{~kg}$ seeds. It increases disease resistance and drought tolerance. It also promotes production of growth promoting substance i.e. 
IAA. The effect has been observed on maize in the region specifically in case of growth contributing characters of the similar findings has been observed by Wagner (2012). The role and importance of biofertizer in sustainable crop production has been reviewed by several authors Biswas et al., (1985), Wani and Lee (1995), Katyal et al., (1994).

\section{Results and Discussion}

\section{Growth attributes of maize}

Amongst various treatments, $\mathrm{T}_{11}$ (Azotobacter + 30kg/ha N (VC) + $60 \mathrm{~kg} / \mathrm{ha} \mathrm{N}$ (urea)] resulted in maximum plant height at 30,60 and 90DAS, followed by $\mathrm{T}_{14}$ [(Azospirillium + $30 \mathrm{~kg} / \mathrm{ha} \mathrm{N}(\mathrm{FYM})+60 \mathrm{~kg} / \mathrm{haN}$ (urea)]. At 90 DAS, the maximum plant height was observed under the treatment $\mathrm{T} 11$ $[($ Azotobacter $+30 \mathrm{~kg} / \mathrm{ha} \mathrm{N}(\mathrm{VC})+60 \mathrm{~kg} / \mathrm{ha}$ $\mathrm{N}$ (urea)] and closely followed by $\mathrm{T}_{9}$ $[($ Azotobacter $+30 \mathrm{~kg} / \mathrm{haN}(\mathrm{PM})+60 \mathrm{~kg} / \mathrm{ha}$ $\mathrm{N}$ (urea)].

During both the years the minimum plant height was recorded in the plot treated with $120 \mathrm{~kg} / \mathrm{ha} \mathrm{N}$ (Urea) which was control. Plant height is mainly decided by the availability of $\mathrm{N}$ nutrient at optimum level, which was met out with the application of $50 \% \mathrm{~N}$ in combination of vermicompost and Azotobacter. Similar results on plant height have also been reported by Bhat et al., (2000).

The various treatments showed nonsignificant effect on number of leaves and stem diameter at 30, 60 and 90 DAS. But the stem diameter was found maximum with the treatment $\mathrm{T}_{11}[$ (Azotobactor $+30 \mathrm{~kg} / \mathrm{ha}$ $\mathrm{N}(\mathrm{VC})+60 \mathrm{~kg} / \mathrm{ha} \mathrm{N}$ (urea)] and it showed better results .It was the result of additional nitrogen supplied by Azotobacter through atmospheric nitrogen fixation.
It was noted that vermi-compost when combined with Azotobacter gave comparable or higher values for stem diameter. Similar findings were also reported by Rohitashav et al., (1993), Elgala et al., (1995) and Mishraet al., (1995).

The maximum plant dry weight was recorded in the plots treated with $\mathrm{T}_{11}$ [(Azotobactor + $30 \mathrm{~kg} / \mathrm{ha} \mathrm{N}(\mathrm{VC})+60 \mathrm{~kg} / \mathrm{ha} \mathrm{N}$ (urea)] during both the years and minimum value was recorded with control plot. The additional supply of nitrogen by Azotobacter could be the reason for maximum plant dry weight. Similar findings were also reported by (Mallic andSaric 1998) and Gaur et al., (2003).

Similar results with the application of vermicompost also have been reported by Patil et al., (1992), Vasanthi and Dravid (1999) and Gondek et al., (2003) (Table 1).

\section{Yield and yield attributes of maize}

As shown in Table 2, yield attributes like cob length, number of grain rows/ cob, number of grains/ row, cob diameter, seed index and yield (quintal / hectare) were significantly increased with the application of $\mathrm{T}_{11}$ [(Azotobactor + 30kg/ha N(VC)+60 kg/ha $\mathrm{N}$ (urea)] during both the years followed by $\mathrm{T}_{9}$ $[($ Azotobacter $+30 \mathrm{~kg} / \mathrm{ha} \mathrm{N}(\mathrm{PM})+60 \mathrm{~kg} / \mathrm{ha}$ $\mathrm{N}$ (urea)], which was statistically found at par with each other. Similar results with the application of bio-fertilizers also have been reported by Rout et al., (2001).

The plots receiving $\mathrm{T}_{0} 120 \mathrm{~kg} / \mathrm{ha} \mathrm{N}$ (urea) which was control, showed the minimum values of yield attributes and yield during both the years of the study because only inorganic fertilizer was applied without any manure and bio-fertilizer. 
Table.1 Plant height, number of leaves at 30, 60 and 90 DAS as influenced by integrated nutrient management

\begin{tabular}{|c|c|c|c|c|c|c|c|c|c|c|c|c|c|c|c|c|c|c|c|}
\hline \multirow[t]{3}{*}{ S.No } & \multirow[t]{3}{*}{ Treatments } & \multicolumn{9}{|c|}{2012} & \multicolumn{9}{|c|}{2013} \\
\hline & & \multicolumn{3}{|c|}{ Plant height (cm) } & \multicolumn{3}{|c|}{ No. of Leaves } & \multicolumn{3}{|c|}{ Plant dry weight(g) } & \multicolumn{3}{|c|}{ Plant height (cm) } & \multicolumn{3}{|c|}{ No. of Leaves } & \multicolumn{3}{|c|}{ Plant dry weight $(\mathrm{g})$} \\
\hline & & $\begin{array}{c}30 \\
\text { DAS }\end{array}$ & $\begin{array}{c}\text { 60 } \\
\text { DAS }\end{array}$ & $\begin{array}{c}90 \\
\text { DAS }\end{array}$ & $\begin{array}{c}\text { 30 } \\
\text { DAS }\end{array}$ & $\begin{array}{c}\text { 60 } \\
\text { DAS }\end{array}$ & $\begin{array}{c}90 \\
\text { DAS }\end{array}$ & $\begin{array}{c}\text { 30 } \\
\text { DAS }\end{array}$ & $\begin{array}{c}\text { 60 } \\
\text { DAS }\end{array}$ & $\begin{array}{c}90 \\
\text { DAS }\end{array}$ & $\begin{array}{c}\text { 30 } \\
\text { DAS }\end{array}$ & $\begin{array}{c}\text { 60 } \\
\text { DAS }\end{array}$ & $\begin{array}{c}90 \\
\text { DAS }\end{array}$ & $\begin{array}{c}30 \\
\text { DAS }\end{array}$ & $\begin{array}{c}\text { 60 } \\
\text { DAS }\end{array}$ & $\begin{array}{c}90 \\
\text { DAS }\end{array}$ & $\begin{array}{c}30 \\
\text { DAS }\end{array}$ & $\begin{array}{c}\text { 60 } \\
\text { DAS }\end{array}$ & $\begin{array}{l}90 \\
\text { DAS }\end{array}$ \\
\hline $\mathbf{T}_{\mathbf{0}}$ & $120 \mathrm{~kg} / \mathrm{ha} \mathrm{N}$ (urea) & 54.0 & 118.9 & 193.2 & 7.3 & 9.0 & 12.7 & 27.0 & 80.8 & 191.6 & 53.8 & 122.2 & 194.8 & 7.3 & 9.4 & 12.6 & 27.2 & 82.1 & 192.0 \\
\hline $\mathbf{T}_{1}$ & $60 \mathrm{~kg} / \mathrm{ha} \mathrm{N}(\mathrm{PM})+60 \mathrm{~kg} / \mathrm{ha} \mathrm{N}$ (urea) & 56.7 & 121.7 & 194.8 & 7.5 & 9.2 & 12.8 & 31.2 & 93.5 & 292.2 & 54.8 & 123.9 & 196.3 & 7.5 & 9.6 & 12.9 & 31.4 & 95.1 & 293.7 \\
\hline $\mathbf{T}_{2}$ & $60 \mathrm{~kg} / \mathrm{ha} \mathrm{N}(\mathrm{FYM})+60 \mathrm{~kg} / \mathrm{ha} \mathrm{N}$ (urea) & 47.8 & 126.5 & 195.3 & 7.5 & 9.2 & 12.7 & 27.3 & 82.3 & 211.4 & 49.9 & 120.2 & 198.9 & 7.2 & 9.6 & 13.0 & 27.8 & 84.1 & 212.5 \\
\hline $\mathbf{T}_{3}$ & $60 \mathrm{~kg} / \mathrm{ha} \mathrm{N}(\mathrm{VC})+60 \mathrm{~kg} / \mathrm{ha} \mathrm{N}$ (urea) & 55.5 & 121.1 & 210.7 & 7.5 & 9.5 & 13.2 & 31.5 & 117.7 & 341.9 & 59.5 & 127.4 & 210.0 & 7.6 & 9.6 & 13.2 & 31.5 & 118.6 & 343.5 \\
\hline $\mathbf{T}_{4}$ & $\mathrm{VAM}+60 \mathrm{~kg} / \mathrm{ha} \mathrm{N}$ (urea) & 57.3 & 117.6 & 197.8 & 7.3 & 9.3 & 12.9 & 28.6 & 83.06 & 288.7 & 57.0 & 124.2 & 199.7 & 7.5 & 9.1 & 13.1 & 28.8 & 84.7 & 289.3 \\
\hline $\mathbf{T}_{5}$ & $\mathrm{VAM}+30 \mathrm{~kg} / \mathrm{ha} \mathrm{N}(\mathrm{PM})+60 \mathrm{~kg} / \mathrm{ha} \mathrm{N}$ (urea) & 52.7 & 121.5 & 202.0 & 7.3 & 9.2 & 12.6 & 27.1 & 81.3 & 207.5 & 53.0 & 121.0 & 204.8 & 7.3 & 9.7 & 12.8 & 28.2 & 83.9 & 207.8 \\
\hline$T_{6}$ & $\mathrm{VAM}+30 \mathrm{~kg} / \mathrm{ha} \mathrm{N}(\mathrm{FYM})+60 \mathrm{~kg} / \mathrm{ha} \mathrm{N}$ (urea) & 58.6 & 120.8 & 208.6 & 7.4 & 9.3 & 12.4 & 30.1 & 102.7 & 222.5 & 55.7 & 123.2 & 209.6 & 7.3 & 9.1 & 12.5 & 30.4 & 104.0 & 223.6 \\
\hline $\mathbf{T}_{7}$ & $\mathrm{VAM}+30 \mathrm{~kg} / \mathrm{ha} \mathrm{N}(\mathrm{VC})+60 \mathrm{~kg} / \mathrm{ha} \mathrm{N}$ (urea) & 56.9 & 118.5 & 207.1 & 7.5 & 9.1 & 13.2 & 32.2 & 118.7 & 351.9 & 60.8 & 123.3 & 211.2 & 7.3 & 9.0 & 13.3 & 32.3 & 120.6 & 352.8 \\
\hline $\mathbf{T}_{8}$ & Azotobacter $+60 \mathrm{~kg} / \mathrm{ha} \mathrm{N}$ (urea) & 55.3 & 119.2 & 205.3 & 7.5 & 9.0 & 13.1 & 30.2 & 117.1 & 211.3 & 57.0 & 121.0 & 208.3 & 7.2 & 9.0 & 13.2 & 31.2 & 118.1 & 213.2 \\
\hline $\mathbf{T}_{9}$ & Azotobacter $+30 \mathrm{~kg} / \mathrm{ha} \mathrm{N}(\mathrm{PM})+60 \mathrm{~kg} / \mathrm{ha} \mathrm{N}($ urea $)$ & 63.3 & 122.0 & 220.2 & 7.5 & 9.8 & 13.5 & 36.7 & 124.9 & 392.4 & 61.9 & 122.8 & 223.5 & 7.5 & 9.3 & 13.5 & 36.8 & 124.6 & 392.6 \\
\hline $\mathbf{T}_{10}$ & Azotobacter $+30 \mathrm{~kg} / \mathrm{ha} \mathrm{N}(\mathrm{FYM})+\mathrm{kg} / \mathrm{ha} \mathrm{N}($ urea $)$ & 58.7 & 123.2 & 215.0 & 7.4 & 9.6 & 12.6 & 29.7 & 93.5 & 243.1 & 58.6 & 124.8 & 211.5 & 7.4 & 9.5 & 12.7 & 30.3 & 94.3 & 245.3 \\
\hline $\mathbf{T}_{11}$ & Azotobacter $+30 \mathrm{~kg} / \mathrm{ha} \mathrm{N}(\mathrm{VC})+60 \mathrm{~kg} / \mathrm{ha} \mathrm{N}$ (urea) & 64.8 & 128.0 & 224.3 & 7.5 & 9.9 & 13.6 & 36.6 & 119.5 & 431.4 & 65.2 & 132.6 & 226.6 & 7.5 & 9.7 & 13.6 & 36.6 & 126.6 & 432.6 \\
\hline $\mathbf{T}_{12}$ & Azospirillium $+60 \mathrm{~kg} / \mathrm{ha} \mathrm{N}$ (urea) & 56.4 & 122.2 & 203.8 & 7.5 & 9.2 & 12.7 & 27.9 & 83.2 & 286.7 & 56.8 & 119.6 & 204.9 & 7.4 & 9.2 & 12.7 & 28.8 & 85.7 & 287.7 \\
\hline $\mathbf{T}_{13}$ & $\begin{array}{l}\text { Azospirillium }+30 \mathrm{~kg} / \mathrm{ha} \mathrm{N}(\mathrm{PM})+60 \mathrm{~kg} / \mathrm{ha} \\
\mathrm{N} \text { (urea) }\end{array}$ & 56.0 & 121.1 & 208.4 & 7.5 & 9.1 & 13.0 & 27.3 & 91.04 & 271.5 & 57.9 & 122.8 & 209.6 & 7.5 & 8.8 & 13.0 & 28.2 & 93.9 & 273.2 \\
\hline $\mathbf{T}_{14}$ & $\underset{\text { Azospirllium }+30 \mathrm{~kg} / \mathrm{ha} \mathrm{N} \text { (FYM) }}{3 \text { (urea) }}+60 \mathrm{~kg} / \mathrm{ha} \mathrm{N}$ & 56.0 & 124.9 & 207.2 & 7.3 & 9.5 & 13.1 & 28.5 & 85.9 & 312.5 & 57.1 & 121.8 & 207.3 & 7.5 & 9.3 & 13.2 & 31.9 & 86.6 & 314.5 \\
\hline $\mathbf{T}_{15}$ & $\begin{array}{c}\text { Azospirillium }+30 \mathrm{~kg} / \mathrm{ha} \mathrm{N}(\mathrm{VC})+60 \mathrm{~kg} / \mathrm{ha} \mathrm{N} \\
\text { (urea) }\end{array}$ & 56.3 & 119.5 & 210.6 & 7.4 & 9.6 & 12.9 & 28.9 & 85.5 & 321.0 & 57.1 & 120.0 & 209.2 & 7.3 & 9.6 & 13.0 & 30.8 & 88.0 & 322.0 \\
\hline & F. Test & NS & NS & $\mathrm{S}$ & NS & NS & NS & $\mathrm{S}$ & $\mathrm{S}$ & $\mathrm{S}$ & NS & NS & $\mathrm{S}$ & NS & NS & NS & $\mathrm{S}$ & $\mathrm{S}$ & $\mathrm{S}$ \\
\hline & C.D (5\%) & 0.26 & 0.33 & 1.47 & 0.21 & 0.63 & 0.45 & 1.61 & 1.94 & 3.01 & 0.24 & 0.32 & 0.49 & 0.17 & 0.35 & 0.54 & 1.36 & 1.43 & 2.30 \\
\hline & S.E. M+_ & 0.12 & 0.16 & 0.70 & 0.10 & 0.30 & 0.22 & 0.77 & 0.93 & 1.45 & 0.11 & 0.15 & 0.24 & 0.08 & 0.17 & 0.26 & 0.65 & 0.68 & 1.11 \\
\hline
\end{tabular}


Table.2 Cob length, no. of grain rows/cob, no. of grains/row, grains/cob and yield as influenced by integrated nutrient management

\begin{tabular}{|c|c|c|c|c|c|c|c|c|c|c|c|c|c|}
\hline \multirow[t]{2}{*}{ S.No } & \multirow[t]{2}{*}{ Treatments } & \multicolumn{6}{|c|}{2012} & \multicolumn{6}{|c|}{2013} \\
\hline & & $\begin{array}{c}\text { Cob } \\
\text { length }\end{array}$ & $\begin{array}{c}\text { Grain } \\
\text { rows/co }\end{array}$ & $\begin{array}{c}\text { Grains/ } \\
\text { row }\end{array}$ & $\begin{array}{c}\text { Cob } \\
\text { diameter(cm) }\end{array}$ & $\begin{array}{l}\text { Seed } \\
\text { Index }\end{array}$ & $\begin{array}{c}\text { Yield } \\
(\mathbf{k g} / \mathbf{h a})\end{array}$ & $\begin{array}{l}\text { Cob length } \\
(\mathrm{cm})\end{array}$ & $\begin{array}{c}\text { Grain } \\
\text { rows/cob }\end{array}$ & $\begin{array}{c}\text { Grains/ } \\
\text { row }\end{array}$ & $\begin{array}{c}\text { Cob } \\
\text { diameter(cm) }\end{array}$ & $\begin{array}{l}\text { Seed } \\
\text { Index }\end{array}$ & $\begin{array}{c}\text { Yield } \\
\text { (kg/ha) }\end{array}$ \\
\hline $\mathbf{T}_{0}$ & $120 \mathrm{~kg} / \mathrm{ha} \mathrm{N}$ (urea) & 14.6 & 12.0 & 27.0 & 3.5 & 20.6 & 4100 & 14.8 & 12.1 & 27.1 & 3.6 & 20.7 & 4280 \\
\hline$T_{1}$ & $60 \mathrm{~kg} / \mathrm{ha} \mathrm{N}(\mathrm{PM})+60 \mathrm{~kg} / \mathrm{ha} \mathrm{N}$ (urea) & 14.9 & 12.1 & 23.2 & 3.6 & 20.7 & 4200 & 15.3 & 12.1 & 27.2 & 3.6 & 21.2 & 4420 \\
\hline $\mathbf{T}_{2}$ & $60 \mathrm{~kg} / \mathrm{ha} \mathrm{N}(\mathrm{FYM})+60 \mathrm{~kg} / \mathrm{ha} \mathrm{N}$ (urea) & 15.0 & 12.2 & 27.5 & 3.7 & 21.3 & 4370 & 15.1 & 12.5 & 27.5 & 3.7 & 21.4 & 4470 \\
\hline $\mathbf{T}_{3}$ & $60 \mathrm{~kg} / \mathrm{ha} \mathrm{N}(\mathrm{VC})+60 \mathrm{~kg} / \mathrm{ha} \mathrm{N}$ (urea) & 17.3 & 15.0 & 30.1 & 4.5 & 24.0 & 5080 & 17.4 & 15.2 & 30.2 & 4.6 & 25.7 & 5250 \\
\hline $\mathbf{T}_{4}$ & VAM + 60 kg/ha N (urea) & 16.2 & 12.9 & 28.4 & 3.9 & 23.1 & 4730 & 16.3 & 12.7 & 28.8 & 4.0 & 24.3 & 4810 \\
\hline$T_{5}$ & $\mathrm{VAM}+30 \mathrm{~kg} / \mathrm{ha} \mathrm{N}(\mathrm{PM})+60 \mathrm{~kg} / \mathrm{ha} \mathrm{N}$ (urea) & 15.1 & 12.2 & 27.9 & 3.8 & 21.2 & 4420 & 15.6 & 12.3 & 27.7 & 3.8 & 21.7 & 4500 \\
\hline$T_{6}$ & $\mathrm{VAM}+30 \mathrm{~kg} / \mathrm{ha} \mathrm{N}(\mathrm{FYM})+60 \mathrm{~kg} / \mathrm{ha} \mathrm{N}$ (urea) & 16.7 & 13.1 & 28.9 & 4.0 & 23.4 & 4760 & 16.6 & 13.1 & 28.9 & 4.0 & 24.8 & 4860 \\
\hline $\mathbf{T}_{7}$ & $\mathrm{VAM}+30 \mathrm{~kg} / \mathrm{ha} \mathrm{N}(\mathrm{VC})+60 \mathrm{~kg} / \mathrm{ha} \mathrm{N}$ (urea) & 17.4 & 15.5 & 30.7 & 4.6 & 24.8 & 5300 & 17.5 & 15.6 & 30.8 & 4.6 & 25.7 & 5390 \\
\hline $\mathbf{T}_{8}$ & Azotobacter $+60 \mathrm{~kg} / \mathrm{ha} \mathrm{N}$ (urea) & 16.7 & 13.2 & 29.4 & 4.0 & 23.8 & 4860 & 16.8 & 13.2 & 29.4 & 4.1 & 25.3 & 4980 \\
\hline $\mathbf{T}_{9}$ & Azotobacter $+30 \mathrm{~kg} / \mathrm{ha} \mathrm{N}(\mathrm{PM})+60 \mathrm{~kg} / \mathrm{ha} \mathrm{N}$ (urea) & 17.8 & 15.7 & 31.6 & 4.8 & 25.6 & 5330 & 17.8 & 15.7 & 31.6 & 4.8 & 26.1 & 5410 \\
\hline $\mathbf{T}_{10}$ & Azotobacter $+30 \mathrm{~kg} / \mathrm{ha} \mathrm{N}(\mathrm{FYM})+\mathrm{kg} / \mathrm{ha} \mathrm{N}($ urea $)$ & 17.1 & 14.0 & 29.9 & 4.5 & 24.4 & 5270 & 17.2 & 14.1 & 30.0 & 4.5 & 25.6 & 5350 \\
\hline$T_{11}$ & Azotobacter $+30 \mathrm{~kg} / \mathrm{ha} \mathrm{N}(\mathrm{VC})+60 \mathrm{~kg} / \mathrm{ha} \mathrm{N}($ urea) & 17.9 & 16.0 & 32.5 & 4.9 & 25.7 & 5360 & 18.0 & 16.0 & 32.7 & 4.9 & 26.4 & 5450 \\
\hline $\mathbf{T}_{12}$ & Azospirillium $+60 \mathrm{~kg} / \mathrm{ha} \mathrm{N}$ (urea) & 16.0 & 12.8 & 28.5 & 3.9 & 22.6 & 4600 & 16.1 & 12.8 & 28.2 & 3.9 & 23.7 & 4770 \\
\hline $\mathbf{T}_{13}$ & $\begin{array}{l}\text { Azospirillium }+30 \mathrm{~kg} / \mathrm{ha} \mathrm{N}(\mathrm{PM})+60 \mathrm{~kg} / \mathrm{ha} \\
\mathrm{N}(\text { urea) }\end{array}$ & 17.4 & 12.7 & 28.2 & 3.9 & 22.1 & 4570 & 15.6 & 12.7 & 28.2 & 3.9 & 23.5 & 4660 \\
\hline $\mathbf{T}_{14}$ & $\begin{array}{l}\text { Azospirllium + } 30 \mathrm{~kg} / \mathrm{ha} \mathrm{N}(\mathrm{FYM})+60 \mathrm{~kg} / \mathrm{ha} \mathrm{N} \\
\text { (urea }\end{array}$ & 15.7 & 12.3 & 28.1 & 3.8 & 22.5 & 4550 & 15.8 & 12.3 & 28.2 & 3.8 & 22.3 & 4560 \\
\hline $\mathbf{T}_{15}$ & Azospirillium $+30 \mathrm{~kg} / \mathrm{ha} \mathrm{N}(\mathrm{VC})+60 \mathrm{~kg} / \mathrm{ha} \mathrm{N}$ (urea) & 15.5 & 12.2 & 28.0 & 3.8 & 21.7 & 4530 & 15.5 & 11.7 & 28.0 & 3.8 & 22.5 & 4540 \\
\hline & F. Test & $\mathbf{S}$ & $\mathbf{S}$ & $\mathbf{S}$ & $\mathbf{S}$ & $\mathbf{S}$ & $\mathbf{S}$ & $\mathbf{S}$ & $\mathbf{S}$ & $\mathbf{S}$ & $\mathbf{S}$ & $\mathbf{S}$ & $\mathbf{S}$ \\
\hline & C.D (5\%) & 0.26 & 0.33 & 1.47 & 0.21 & 0.63 & 0.45 & 0.24 & 0.32 & 0.49 & 0.17 & 0.35 & 0.54 \\
\hline & S.E. Mean+_ & 0.12 & 0.16 & 0.70 & 0.10 & 0.30 & 0.22 & 0.11 & 0.15 & 0.24 & 0.08 & 0.17 & 0.26 \\
\hline
\end{tabular}




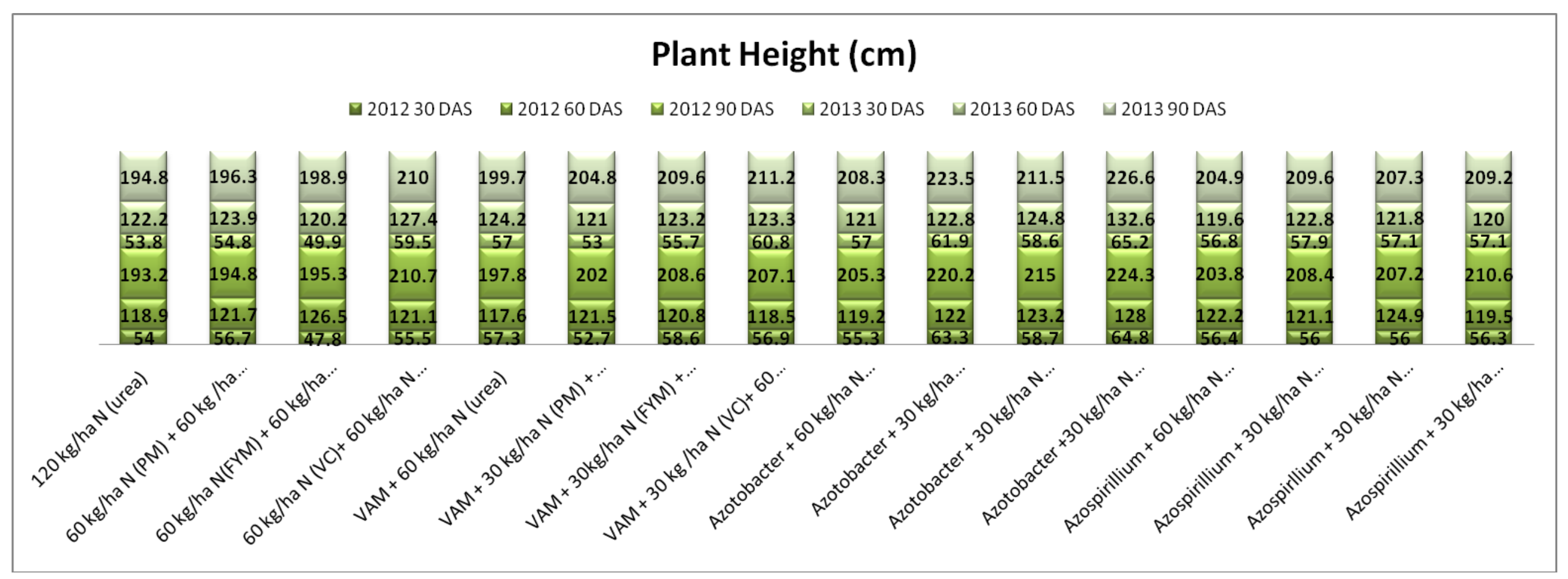




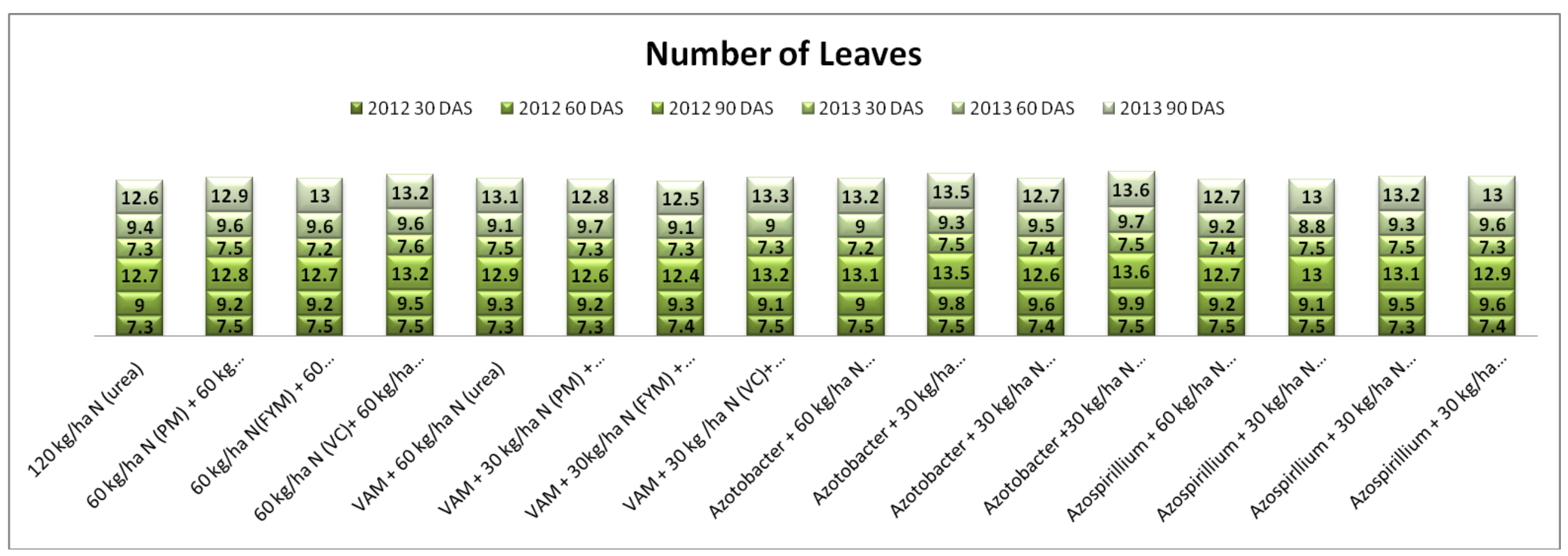




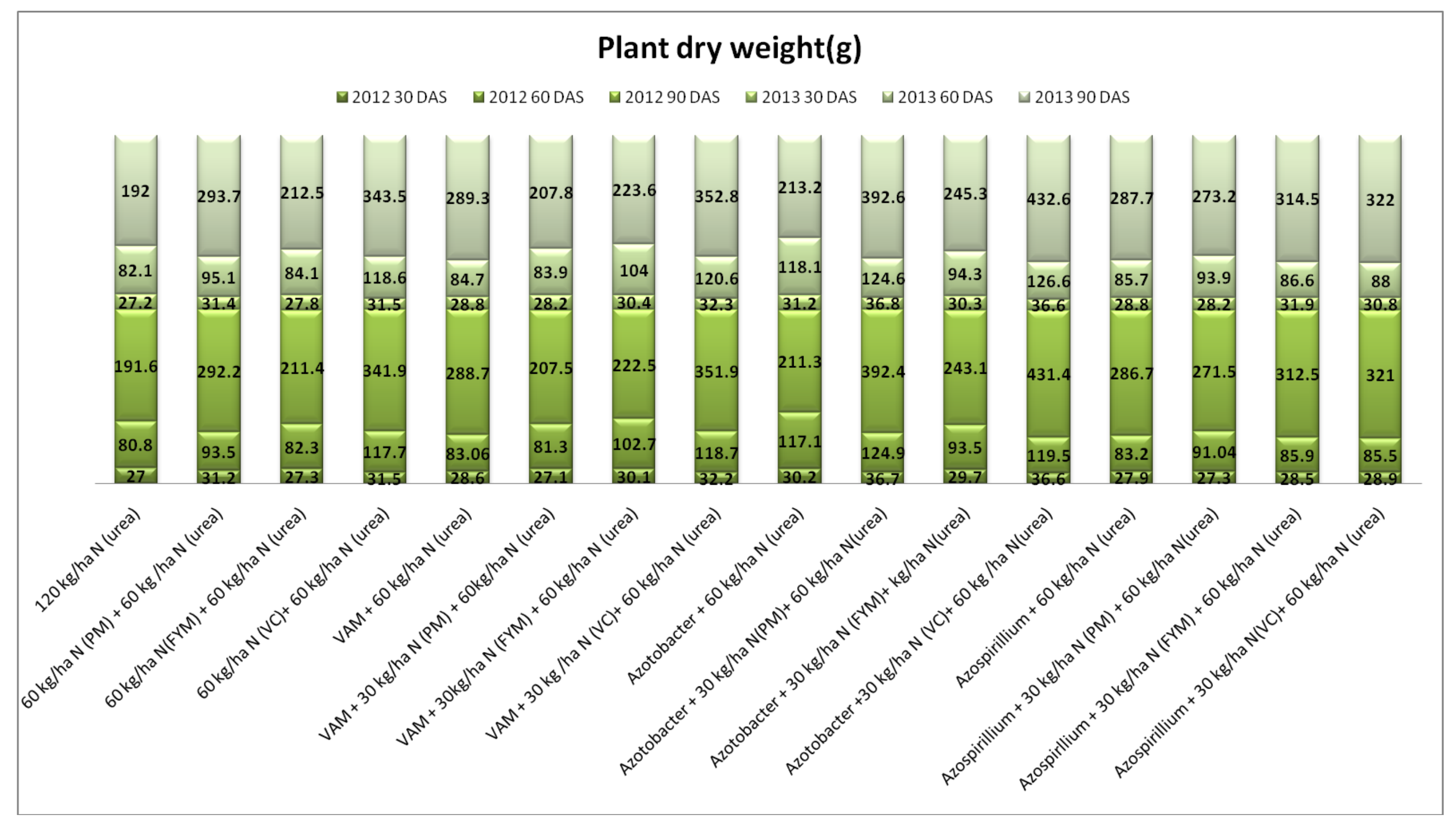


The increase in cob diameter might be due to integrated application of vermicompost and chemical fertilizer compared to the other treatments. The best results in terms in crop yield and yield attributes were obtained with vermi- compost and $50 \%$ chemical fertilizer. Similar findings were supported by the results of Das et al., , (2002).

\section{Acknowledgements}

The authors are thankful to the Principal Dayanand college Ajmer (affiliated to Swami Keshwanand Agricultural University, Bikaner) for giving permission and help during the entire field experiment.

\section{References}

Bhat, M.D., Singh,K.N., Bali, Amarjit and Shah,M.H.(2000). Grain yield of oat as influenced by sowing time and nitrogen levels under temperate conditions of Kashmir . Indian Journal of Agronomy, 45(1) : 199-204.

Biswas,B.C.Yadav,D.S., and Satish Mahashwari,1985.Biofertilizers in Indian Agriculture.Fertilizers news. 30(10) : 20-28.

Das, P.K., Jena, M.K. and Sahoo, K.C.(2002). Effect of integrated application of vermicompost and chemical fertilizer on growth and yield of paddy in red soil of South Eastern ghats zone of Orissa. Environment and Ecology 20(1): 13-15.

Elgala, A., Ishac, Y.Z., Abdel, Monem , M., Huang ,P.M., Bethelin , J.andBollag, J.M. (1995). Effect of single and combined inoculation with Azotobacter and VAM fungi on growth and mineral nutrient contents of maize and wheat plants. Inorganic and microbial activities, 14(2): 109116.

Gaur,N.K., Sharma, O.P.,Bhati, T.K., Narain,
P., kathju,S., Singh, M.P. and Praveen, Kumar (2003). Effect of fertility levels and Azotobacter inoculation on yield attributes and yield of barley. Human impact on desert environment, 329332.

Gondek, K., Filipek and Mazur, B.(2003).Biomass yields of shoots \& roots of plants cultivated amended with vermicompost nutrient contents of maize and wheat plants. Environmental impact of solid component interactions. Inorganic and microbial activities, 14(2): 109-116.

Katyal,J.C.Venkatashwarlu,B., and Das S.K.1994.Biofertilizer for Nutrient supplementation in Dryland Agriculture.Fertilizer News. 39(4): 2732.

Kumar,V.and Singh A.P.(1999). Integrated use of fertilizers and FYM in rabi maize. Annual of Plant and Soil Research, 4(1):189-190.

Lamani, C.S., Palled, Y.B. and Shenoy, H (2000). Soils organic carbon as influenced by integrated nutrient management in hybrid maize. Current Research University Agricultural Sciences Bangalore, 29 (3-4) : 37-38.

Mallic,V. ans , M.R.(1998). Effectiveness of Azotobacter in relation to maize genotype and the nitrogen content of nutrient solution. Microbiologija Beograd, 25(1): 45-46.

Mishra,O.R., Tomar, U.S.,Sharma, R.A. and Rajput, A.M.(1995). Response of maize to chemicals and bio-fertilisers. Crop Research Hisar, 9(2): 233-237.

Rohitashav,Singh.,Sood,B.,Sharma,V.K. and Singh,R. (1993).Response of forage maize(Zea mays) to Azotobacter inoculation and nitrogen. Indian Journal of agronomy, 15(2): 53-55.

Rout,D., Satapathy, M.R. and Mohanpatra, B.K.(2001). Effect of biofertiliser inoculation and growth and yield of 
maize. Madras Agricultural Journal, 88 (7-9), 530- 532.

Vasanthi,D. and Kumaraswamy, K.(1999).Efficacy of vermicompost to improve soil fertility and rice yield. Journal of the Indian Society of Soil Science, 47(2): 268-272.

Virmani,S.M.(1994).UNC TAD agenda21.The new challenges for soils research. Journal Indian Society of Soil Science, 42(5): 16-24.

Wani,S.P.and Lee, K.K.1995.Microorganisms as biological inputs for sustainable agriculture in organic Agriculture (Thampan,P.K.ed.) Peekay Tree Crops Development Foundation, cochin,India, pp- 39-76.

\section{How to cite this article:}

Rubina Gill, Prasoon Singh' Rajesh Kumar, Bijendra Kumar. 2018. Effect of Integrated Nutrient Management on Plant Growth and Yield of Rabi Maize under Irrigated Conditions of Ajmer. Int.J.Curr.Microbiol.App.Sci. 7(03): 2103-2112. doi: https://doi.org/10.20546/ijcmas.2018.703.247 